\title{
Un cadre d'analyse des compromis adoptés par les éleveurs pour organiser leur travail ${ }^{*}$
}

\author{
Cécile Fiorelli ${ }^{1}$ \\ Benoît Dedieu ${ }^{1}$ \\ Jocelyne Porcher ${ }^{2}$ \\ ${ }^{1}$ Inra SAD \\ UMR 1273 Métafort \\ Centre Inra de Theix \\ 63122 Saint Genes \\ Champanelle \\ France \\ $<$ fiorelli@clermont.inra.fr> \\ <benoit.dedieu@clermont.inra.fr> \\ 2 Inra SAD \\ UMR Sadapt \\ 16 , rue Claude Bernard \\ 75005 Paris \\ France \\ <porcher@agroparistech.fr>
}

Tirés à part : C. Fiorelli

\begin{abstract}
Résumé
Pour penser des réorganisations du travail respectant le rapport au travail des éleveurs, il faut pouvoir caractériser les facteurs concrets de l'organisation potentiellement en jeu dans une réorganisation, mais aussi ce rapport au travail. À partir d'une articulation théorique des approches sur le travail de la zootechnie et de la psychodynamique et d'une étude de cas chez des éleveurs pluriactifs, nous avons construit un cadre d'analyse de l'organisation du travail. Il permet d'analyser cette organisation comme un compromis entre des attentes vis-à-vis du travail et des contraintes temporelles. Il est constitué de trois entités : les attentes vis-à-vis du travail, représentées sous forme de cinq rationalités subjectives, les contraintes horaires et calendaires et quatre facteurs de l'organisation du travail déterminant ce qu'il y a à faire, les gens et le temps pour le faire. Chacun des quatre facteurs de l'organisation du travail est analysé selon un double point de vue : à quelles rationalités subjectives du travail et à quelles contraintes temporelles renvoient-ils? Après avoir décrit le cadre d'analyse, nous présentons son application à deux cas.
\end{abstract}

Mots clés : agriculture à temps partiel ; ferme d'élevage ; organisation du travail.

Thèmes : économie et développement rural ; méthodes et outils ; productions animales.

\section{Abstract \\ Proposal for an analytic framework of the compromises adopted by livestock farmers to improve their work organisation}

Improving the work organisation of livestock farmers requires understanding both the concrete components of work organisation and the feelings of farmers about their work. Based on a theoretical study of the work approaches developed by animal sciences and psychodynamics science and on a multiple-job-holder case study, we developed an analytic framework of the work organisation on a livestock farm. This framework made it possible to analyse this work organisation as a compromise between farmers' expectations about their work and their time constraints. It was composed of three entities: farmers' expectations about their work, represented by the combination of five types of subjective rationalities of work, time and calendar constraints and four factors of work organisation determining the work to be done, the people involved and the time to do it. Each factor of the work organisation was analysed according to two points of view: to what subjective work rationalities and to what time constraints does each factor refer? After describing the analytic framework, we present its application to two cases to reveal the power of this new approach to help in clarifying what compromises are made by farmers to cope with work and how they feel about it.

Key words: livestock farms; part time farming; work organization.

Subjects: animal productions; economy and rural development; tools and methods.

*Pour citer cet article: Fiorelli C, Dedieu B, Porcher J. Un cadre d'analyse des compromis adoptés par les éleveurs pour organiser leur travail. Cah Agric 2010; 19: 383-90. DOI: 10.1684/agr.2010.0424 
E n France, depuis quelques années, en élevage, la maîtrise ou la diminution du temps de travail, la possibilité d'avoir du temps libre pour sa famille, sont des souhaits très fortement exprimés par les agriculteurs lors d'enquêtes (Seegers et al., 2006). Les démarches d'accompagnement qui cherchent à satisfaire ces revendications se multiplient (Boissier, 2006; KlingEveillard, 2008). Les zootechniciens ont mis au point des méthodes d'évaluation des temps de travaux et de la marge de manouvre temporelle (Dedieu et al., 2000), avec une vision du travail centrée sur ses dimensions techniques et temporelles. Ils proposent et évaluent des choix techniques et des formes d'organisation, selon leurs impacts sur la production animale, la productivité de la main-d'œuvre, les durées et rythmes associés à la mise en œuvre des activités (Fagon et Hostiou, 2009). Cependant, travailler ne met pas seulement en jeu des aspects techniques, temporels ou économiques, cela engage aussi l'identité, les sentiments (Porcher, 2001), la morale (Mouret, 2007)... Le rapport subjectif au travail est quelque chose de personnel et complexe. Macombe (2007) a montré que certaines pratiques, exigeantes en temps ou pénibles physiquement, sont intouchables dans une réorganisation du travail parce qu'elles sont porteuses de l'éthique de métier. On peut donc penser que l'organisation du travail relève de considérations non seulement de durée ou de productivité, mais aussi identitaires, affectives, éthiques.

La mise en relation d'éléments marquants du rapport au travail des éleveurs, d'une part, et de l'organisation, d'autre part, est actuellement absente des méthodes de diagnostic et de conseil utilisées en France dans le secteur de l'élevage (Collectif, 2009). Pourtant les études récentes portant sur les démarches d'accompagnement des éleveurs (Boissier, 2006 ; Kling-Eveillard, 2008) mettent en évidence le besoin d'une telle approche globale. Ses fondements conceptuels restent à élaborer : il s'agit de relier deux approches, celle de l'organisation du travail centrée sur l'élaboration de la production, du temps et du calendrier de travail (Dedieu et al., 2006) et celle du rapport subjectif au travail (Dejours, 1998). L'objectif de cet article est de résumer les bases conceptuelles principales de la construction d'un cadre d'analyse du travail en élevage articulant ces deux approches : l'une, technique et temporelle, et l'autre, subjective, et d'en illustrer le caractère heuristique avec l'analyse de l'organisation du travail dans deux exploitations d'élevage ovin.

\section{Méthode \\ de construction du cadre d'analyse}

La construction du cadre d'analyse de l'organisation du travail en élevage, qui vise à associer les deux approches mentionnées, a consisté à identifier les éléments structurants de l'organisation du travail faisant sens pour l'éleveur.

La construction du cadre a impliqué :

- une réflexion théorique mobilisant les travaux de caractérisation de l'organisation du travail menés en zootechnie des systèmes d'élevage et ceux de psychodynamique du travail analysant les relations entre le sujet et son travail ; - une étude empirique chez un groupe d'éleveurs ovins pluriactifs.

Dans l'approche développée par les zootechniciens, l'organisation du travail est exprimée comme l'agencement, à l'échelle d'une campagne agricole, entre la conduite d'élevage, les bâtiments et les équipements, la main-d'œuvre (Madelrieux et al., 2009). Le dimensionnement des activités et le temps de travail font partie des éléments du contexte ou du projet que l'organisation vise à satisfaire (efficacité, temps disponible pour faire autre chose, temps contraint par une autre activité).

Dans les approches de psychodynamique du travail, le travail ne sert pas qu'à produire et à gagner de l'argent, il permet de se sentir vivant, de se construire en tant qu'homme (Dejours, 1998). Ces travaux soulignent que travailler met en jeu l'identité, le vivre ensemble, les sentiments, les valeurs des personnes et non pas seulement de l'argent ou du temps. Le rapport subjectif au travail, ce que travailler met en jeu pour chaque personne, le sens que donne la personne au travail, n'est donc pas seulement d'ordre économique ou technique mais aussi d'ordre identitaire, relationnel, moral. Selon que l'organisation du travail respecte, nourrit ou, au contraire, altère le sens donné au travail par la personne, travailler peut générer du plaisir ou au contraire de la souffrance (Dejours, 1998).

L'étude empirique a porté sur huit exploitations d'élevage ovin gérées par des pluriactifs dans le Puy-de-Dôme (France). Il s'agit d'une étude de cas instrumentale, privilégiant de fait un faible nombre et une diversité de cas, ainsi qu'une analyse approfondie de chaque cas (David, 2004). Les cas ne sont pas étudiés pour euxmêmes, c'est-à-dire pour analyser spécifiquement le travail des éleveurs ovins pluriactifs du Puy-de-Dôme, mais à titre d'exemples facilitant la mise en évidence des relations entre organisation du travail, rapport subjectif au travail et contraintes temporelles. En effet, la pluriactivité exacerbe, d'une part, l'importance et la diversité des contraintes qui pèsent sur l'organisation du travail en termes de durée et de complexité d'articulation temporelle des activités (Dedieu et al., 1999) et, d'autre part, la diversité des motivations économiques et non économiques des pluriactifs pour mener une activité agricole (loisir, relations à la nature, tradition familiale... [Barlett, 1986 ; Bessant, 2000]).

Sur la base d'une étude antérieure (Fiorelli et al., 2007a), nous avons choisi des cas de façon à couvrir une diversité : - de fonctionnements de l'élevage (conduite de la reproduction et de la commercialisation);

- d'attentes de revenu (de travail à perte accepté à revenu majoritaire ou indispensable pour le ménage) ;

- et de tensions sur le temps de travail (taille du troupeau ovin, durée et rythmes de l'activité non agricole).

Trois entretiens d'environ deux heures chacun ont été réalisés dans chaque ferme. Tous les entretiens ont été traités en analysant le discours et en complétant pas à pas les monographies. Le premier entretien, de type compréhensif, était réalisé auprès de chaque membre pluriactif du couple (11 personnes pour les 8 fermes), et s'intéressait aux représentations de l'élevage (Sens et Soriano, 2001). Il a permis de construire la grille de caractérisation du rapport subjectif au travail en élevage (Fiorelli et al., 2007b). Les deux autres entretiens étaient réalisés de manière semi-directive auprès du chef d'exploitation: l'un sur les pratiques d'élevage (reproduction, alimentation, gestion sanitaire, des mises bas, allotement), l'autre sur le "qui fait quoi", à partir de la reconstitution de journées types couvrant toute l'année. Ces deux 
entretiens ont permis d'élaborer la grille de caractérisation de l'organisation du travail. Le cadre d'analyse articule ces deux grilles. Il a été construit à partir des monographies, de façon itérative, cas après cas, pour prendre en compte les spécificités de chaque cas.

Le cadre d'analyse vise à identifier les éléments structurants de l'organisation du travail faisant sens pour les éleveurs, pour expliciter les compromis ou les tensions vécues par les éleveurs dans l'organisation du travail. Comme tout modèle conceptuel, notre cadre d'analyse doit être validé. La validation consiste à vérifier qu'il permet d'analyser d'autres cas d'éleveurs et à évaluer ses qualités compréhensives et heuristiques (Coquillard et Hill, 1997), c'est-à-dire ses capacités à produire des connaissances nouvelles et à être un support de conception d'outils d'enseignement ou de conseil. Les qualités en question seront mises en évidence par le fait que des utilisateurs des mondes de la recherche, de l'enseignement ou du développement manifestent de l'intérêt pour ce cadre et s'en emparent. Cette phase de validation n'est, à l'heure actuelle, qu'ébauchée. Nous y reviendrons dans la discussion.

\section{Description du cadre d'analyse}

En identifiant les éléments structurants de l'organisation du travail, le cadre d'analyse vise à expliciter les compromis et les éventuelles tensions dans cette organisation. Il repose sur l'idée que les attentes, économiques et non économiques ainsi que les contraintes temporelles sont structurantes de l'organisation.

Le cadre est constitué de trois sortes d'entités que sont les contraintes temporelles, les attentes vis-à-vis de l'élevage et l'organisation du travail et il les met en relation (figure 1).

Les contraintes temporelles vis-à-vis de l'élevage sont les plages horaires et calendaires pendant lesquelles l'éleveur n'est pas disponible pour l'élevage.

Les attentes vis-à-vis de l'élevage sont identifiées à partir de la caractérisation du rapport subjectif au travail en élevage. Mais comme ce rapport est complexe et personnel, nous avons proposé une caractérisation simplifiée qui consiste à le décomposer en cinq types de rationalités pour la mobiliser dans l'analyse de l'organisation du travail (Fiorelli, 2007b).
Ces cinq types sont : la rationalité économique, technique, identitaire personnelle et professionnelle, relationnelle avec les gens et les animaux et relative à l'engagement du corps au travail. Ce dont rendent compte ces cinq types de rationalités et les éléments du discours ou des pratiques permettant de les identifier sont détaillés dans le tableau 1. La caractérisation consiste à recenser les expressions relatives aux cinq types de rationalités du travail dans les discours des éleveurs sur le travail et leurs pratiques, puis à analyser la manière dont certaines rationalités sont mises en lien entre elles ou en avant par l'éleveur.

L'organisation du travail est représentée par quatre facteurs : le dimensionnement des activités, la composition du collectif de travail et la répartition des tâches, la conduite d'élevage, la gestion du temps dédié à l'élevage. Les éléments de définition et de caractérisation des facteurs sont précisés dans le tableau 2.

L'analyse est centrée sur les relations entre tout ou partie de chacune de ces 3 entités. Pour cela, nous proposons d'identifier à quelles rationalités du travail et à quelles contraintes temporelles le contenu des facteurs renvoie. L'analyse de ces relations permet ainsi d'identifier les éléments struc-

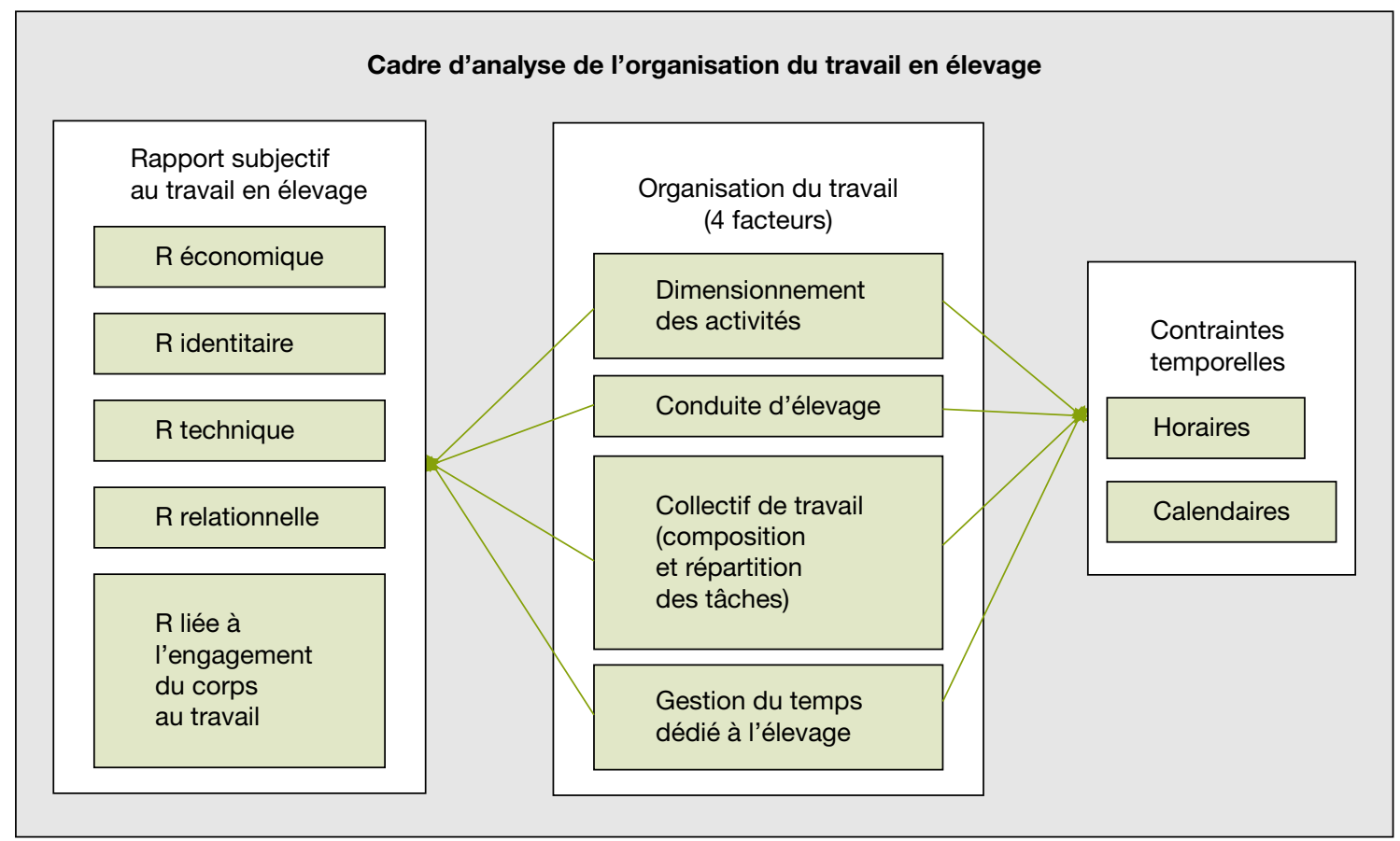

Figure 1. Représentation du cadre d'analyse de l'organisation du travail en élevage.

Figure 1. Representation of the analytic framework of livestock farmer work organisation.

$\mathrm{R}$ : rationalité. 
turants et des tensions ou compromis éventuels dans l'organisation du travail.

\section{Mobilisation}

\section{du cadre pour analyser l'organisation du travail chez deux éleveurs pluriactifs}

Pour illustrer la mobilisation du cadre et sa capacité à mettre en évidence des relations différentes entre organisation du travail, rapport au travail d'élevage et contraintes temporelles, nous avons choisi de montrer l'analyse de l'organisation du travail d'élevage dans deux exploitations conduites par des pluriactifs, parmi les huit ayant servi à l'élaboration du cadre.

Georges, 50 ans, trie le courrier à la Poste 2 nuits toutes les 4 nuits, depuis 25 ans. Sa femme est salariée, elle n'intervient pas sur l'exploitation. Ils n'ont plus d'enfant à charge. Il y a 10 ans, Georges a repris l'exploitation de son beau-père, après avoir travaillé avec lui pendant 15 ans. Il élève 265 brebis sur 35 hectares.

Ses contraintes horaires sont liées au fait qu'il part travailler à la Poste à 19 heures et rentre à 7 heures du matin, qu'il dort une partie de la matinée et qu'il aime être disponible le soir quand il ne travaille pas à la Poste pour être avec sa femme ; ils ont des activités de loisir au moins une fois par semaine. Ses contraintes calendaires correspondent aux 5 semaines minimum de vacances par an pendant lesquelles il quitte l'exploitation.

Georges dit que son travail à la Poste, est un travail "alimentaire", répétitif, sans marge de manouvre, qui "apporte peu de satisfaction ", qui "laisse beaucoup de temps", et apporte un bon revenu. Pour Georges, "l'élevage, c'est un plus dans [sa] vie en général". Il était content de devenir "le patron". Il aime pouvoir prendre des initiatives et "[être] responsable de ses résultats et du temps qu'il lui reste ", ce qui n'est pas possible dans son travail salarié. L'élevage rapporte un revenu complémentaire pour le ménage mais Georges n'en connaît pas le montant. Pour maintenir son train de vie, et en particulier ses vacances et ses voyages, Georges dit qu'il ne peut pas arrêter son travail à la Poste. Il dit

\section{Tableau 1. Description des cinq types de rationalités du travail.}

Table 1. Description of the five types of work rationalities.

\begin{tabular}{ll}
\hline $\begin{array}{l}\text { Rationalité } \\
\text { du travail }\end{array}$ & Ce dont la rationalité du travail rend compte \\
& Éléments de caractérisation dans le discours de l'éleveur et dans ses pratiques
\end{tabular}

économique

Attentes en termes de revenu (accepter de travailler à perte, ne pas perdre d'argent, obtenir un revenu indispensable, tel niveau de revenu...).

Argent: aspects quantitatifs et qualitatifs du revenu, des investissements, de la capitalisation, des besoins du ménage, des coûts, des résultats économiques.

technique

Volonté de maîtrise du processus de production, d'obtention de performances de production et d'efficacité de l'organisation (niée, présente ponctuellement, omniprésente).

Maitrise technique et organisationnelle du processus de production : techniques mises en jeu, savoir-faire, performances techniques, aptitudes des animaux et des surfaces, caractéristiques techniques du matériel, temps de travail, efficacité de l'organisation.

relationnelle

Investissement affectif avec les personnes ou avec les animaux.

Relations entre l'éleveur et les animaux, l'éleveur et sa famille, l'éleveur et ses collègues de travail: I'affectivité, I'amour, la familiarité, la réciprocité, la reconnaissance, les échanges, le partage ou au contraire l'indépendance, le fait d'être seul.

identitaire

Rôle joué par l'élevage pour l'éleveur : exercice et développement de sa liberté, de son intelligence, de sa personnalité et/ou inscription dans un champ professionnel.

- identité dite "personnelle ": être libre, être un grand propriétaire, être sensible, être capable d'apprendre, être indispensable, être particulier, être utile, être responsable, être un maillon d'une histoire familiale, en lien avec l'origine culturelle, géographique ;

- identité "professionnelle " : par exemple, le fait d'être un "bon " éleveur, d'être un "vrai " éleveur, un éleveur "technique", "moderne".

\section{relative à l'engagement du corps au travail}

Façon dont le corps est engagé dans le travail

Diversité des sollicitations, sollicitations contraintes, forcées, intensité des sollicitations, pression de cadence de travail, ou au contraire fait de travailler à son rythme, contact physique avec les animaux, I'environnement de travail (dehors/dedans, la nature/la ville), façon dont les sens sont mobilisés dans le travail (bruits, odeurs), ressenti du travail (sentiment de détente, de fatigue, de pénibilité). 
Tableau 2. Description des quatre facteurs de l'organisation du travail.

Table 2. Description of the four factors to describe the work organisation.

\begin{tabular}{ll}
\hline Facteur & Description \\
\hline Dimensionnement & II concerne le raisonnement des dimensions des activités qui font sens dans l'organisation \\
des activités & du travail d'élevage. Les activités non économiques, dites privées ou de loisirs, si elles jouent \\
& un rôle dans l'organisation du travail, sont à considérer.
\end{tabular}

Caractéristiques : taille du cheptel pour l'activité agricole, taux d'emploi pour les activités salariées, durée annuelle ou nombre des chantiers pour les activités indépendantes ou les activités non économiques, au moment de l'entretien et tout élément parlant de la dynamique des dimensions des différentes activités.

\section{Composition du collectif de travail et répartition des tâches}

\section{Conduite d'élevage}

\section{Gestion du temps}

C'est l'ensemble des travailleurs qui interviennent sur l'exploitation, quels que soient le statut et la fréquence d'intervention.

Caractéristiques : taille du collectif (nombre de personnes), caractère du collectif de travail (familial, amical, voisinage, professionnels rémunérés) ; répartition des tâches liées au travail quotidien avec les bêtes et le travail non quotidien sur les surfaces entre les membres du ménage, de la famille et ceux n'appartenant ni au ménage, ni à la famille, selon ce qui fait sens.

C'est tout ou partie des pratiques d'élevage (reproduction, alimentation, commercialisation) selon le problème à traiter.

Caractéristiques: contenu technique de la pratique d'élevage.

Elle est caractérisée pour une période selon le problème à traiter : la campagne, une période infra-annuelle, des journées types. Sont considérés a priori trois "temps ": le temps " agricole ", le temps "pour les autres activités», le temps apparent de "non-travail » correspondant respectivement au travail agricole, aux autres activités économiques et aux activités privées ; même si, sur le plan subjectif, le travail ne s'arrête pas aux horaires des activités (Dejours, 1993). L'important est d'identifier les temps qui font sens pour l'éleveur dans l'organisation du travail d'élevage...

Caractéristiques : relations entre les temps, temps borné ou illimité, horaires fixes, cadence de travail imposée, subordination des temps. que grâce à l'élevage, il se sent bien, " surle plan physique et tout ", en se comparant à ses collègues de la Poste qui ne font plus grand-chose de leurs journées au même âge.

$\rightarrow$ Les éléments marquants du rapport subjectif au travail en élevage de Georges sont la dominance des rationalités identitaires et techniques: faire de l'élevage, pour Georges, c'est pouvoir exercer sa liberté, son intelligence, être indépendant ; c'est se prouver et prouver aux éleveurs à temps plein qu'il est capable d'obtenir de bonnes performances de production. Il y a une très forte opposition entre l'élevage et son travail de salarié à la Poste, notamment sur le plan identitaire. Sur le plan économique, l'élevage rapporte un revenu inconnu et non indispensable.

Georges a saisi des opportunités, qui lui assurent de bonnes relations dans son voisinage et sa belle-famille, pour augmenter le troupeau et les surfaces dans la limite de l'autonomie fourragère et de la possibilité d'assurer seul le travail sur le troupeau. Georges reconnaît qu'il s'est ainsi donné beaucoup de travail, et qu'il n'en faut pas plus.

$\rightarrow$ Le dimensionnement renvoie aux rationalités techniques, identitaires et relationnelles mais aussi au temps limité que Georges soubaite consacrer à l'élevage.

Georges met en effet un point d'honneur à assurer seul le travail sur le troupeau, notamment dans les périodes critiques que sont les agnelages. En revanche, il n'hésite pas à payer pour se faire remplacer pour partir en vacances et pour soustraiter la mise en balle ronde du foin (Georges peut partir l'esprit tranquille travailler à la Poste le soir après avoir fané), le compostage, l'épandage. Le collectif de travail se compose donc d'un seul membre permanent (lui), de personnes qui interviennent régulièrement, soit pour le remplacer quand il part en vacances (son beau-père ou une personne du village), soit pour réaliser des travaux sur les surfaces (voisin agriculteur et entreprises de travaux agricoles), de personnes qui interviennent une fois par an pour la tonte (le tondeur et un collègue de la Poste pour attraper les brebis).

$\rightarrow$ La composition du collectif de travail et la répartition des tâches renvoient aux rationalités identitaires et relationnelles ainsi qu'aux contraintes boraires de la Poste et calendaires que Georges se donne.

Georges met aussi un point d'honneur à avoir un troupeau en bon état, à satisfaire les demandes du groupement de producteurs en termes de qualité des carcasses et de périodes de vente pour se prouver et prouver aux autres agriculteurs qu'il est un bon éleveur, même en étant pluriactif. Il élève des agneaux de races à viande, plus fragiles mais mieux conformés. Il ne regarde pas à la dépense en concentrés pour engraisser les agneaux. Il commercialise l'ensemble de ses agneaux dans les filières de qualité. S'il fait des impasses, ce n'est pas sur le troupeau, c'est sur l'entretien des surfaces. 
$\rightarrow$ Les choix de conduite du troupeau renvoient surtout aux rationalités identitaires et techniques, tandis que ceux concernant les surfaces renvoient surtout aux contraintes temporelles.

À l'échelle de la journée, son travail d'élevage s'organise surtout entre les horaires de travail à la Poste et de ses activités autres. Le travail agricole est borné le soir. Ponctuellement, Georges peut se faire remplacer à la Poste par des collègues, par exemple pour être moins fatigué pendant le pic d'agnelage où il assure beaucoup de surveillance et de soins. Les projets de voyages à l'étranger sont structurants des calendriers de reproduction et de commercialisation. Pour les vacances plus courtes en France, c'est l'inverse: Georges les cale dans des périodes où il n'y a que du travail avec les animaux et que ce travail est facile à sous-traiter.

$\rightarrow$ La gestion du temps renvoie à la fois aux rationalités techniques et identitaires de l'élevage, et aux contraintes temporelles de Georges.

Pour résumer, les enjeux identitaires et techniques dans le travail d'élevage en termes d'estime de soi, de reconnaissance par les voisins agriculteurs à temps plein et d'obtention de performances de production selon les critères des groupements de commercialisation, ainsi que la volonté d'avoir du temps libre pour les loisirs et les vacances, sont très structurants de l'organisation du travail de Georges. Les choix de conduite renvoient ainsi pour la plupart des modalités aux rationalités identitaires et techniques, à l'exception du choix des périodes de lutte qui renvoie aussi aux contraintes calendaires. La sous-traitance d'une grosse partie du travail sur les surfaces et le remplacement sont raisonnés dans ce sens et non en termes de coût. Le compromis porte donc surtout sur le niveau de revenu agricole. Globalement, Georges est satisfait avec cette organisation.

Béatrice, 40 ans, sans enfant, élève 340 brebis avec Robert, 60 ans. Ils exploitent 90 hectares dont 70 hectares d'herbe et de luzerne et 20 hectares de blé et de tournesol. Pour avoir un statut social indépendant de Robert (elle n'est pas déclarée sur l'exploitation), Béatrice a choisi un travail de mise en rayon au supermarché, 6 jours par semaine de 5 heures à 10 heures, "qui laisse $d u$ temps la journée pour les brebis ", "ses filles". Robert a développé auprès des communes une activité d'entretien de voirie : réalisée dans les périodes creuses de l'activité agricole, elle apporte un revenu complémentaire et ne génère pas de contrainte calendaire. Les contraintes horaires sont liées aux horaires de travail au supermarché de Béatrice et à la nécessité pour elle de se coucher tôt. Il n'y a pas de contrainte calendaire.

Pour Robert et Béatrice, l'élevage a une forte dimension affective : les brebis, ce sont "leurs bébés". Robert explique qu' "aimer les bêtes, ça rapporte", que "les bêtes rendent ce que vous leur donnez". Béatrice raconte: "On ne voit pas les bêtes comme des sous quoi, bon. Can'a rien à voir. Nous, on a du mal à vendre des agneaux alors qu'il y en a qui disent bo ben tient, je vais les faire partir ceux là [...]. " Même s'ils partagent ce fort investissement affectif, Béatrice explique que l'élevage ne met pas en jeu les mêmes choses pour elle et pour Robert : "Moi, les bêtes elles sont bien, moi ça me va. Que l'agneau ça soit un PO, RO, un R+, un $R$ machin que je n'y comprends rien et que je m'en fous, je... Non, je comprends des fois, il reçoit les factures, [...] il râle par rapport, parce qu'il a aussi un roulement d'argent à faire pour payer... Mais moi... Ce n'est pas un truc qui me tracasse. L'argent sur les bêtes, non. Je râlerais que les blés ils les paient tant [...]. Pour moi, [les agneaux], ils sont tous beaux, ils sont tous bien" ; "[...] moi je ne serais pas objective si c'était mon gagne-pain be, je pense que j'aurais fait faillite be depuis longtemps. Parce que... je n'aurais pas... d'abord j'enverrais les agneaux ils auraient bien un an". Robert attend de l'exploitation son revenu principal, mais il n'est pas dans l'idée de maximiser son revenu. Robert insiste sur son intérêt à "être astucieux pour économiser du temps [et réduire l'astreinte] et la pénibilité même si ça coûte un peu plus cher".

$\rightarrow$ Les éléments marquants du rapport subjectif au travail en élevage de Béatrice sont la rationalité relationnelle avec les animaux et la négation des rationalités techniques et économiques. Pour Robert, toutes les rationalités semblent importantes, même si l'économie passe au second plan par rapport à toutes les autres quand il s'agit d'améliorer les performances techniques (limiter la mortalité avec les biberons et une gestion sanitaire intensive) ou de réduire l'astreinte ou la pénibilité du travail (équipement pour distribuer l'alimentation en self-service pour les brebis, eau courante dans les parcs).

Quand Béatrice revient de son travail au supermarché à $10 \mathrm{~h}$ 30, ils partent s'occuper ensemble des animaux "sans regarder [leur] montre, pas comme au supermarché" et repoussent l'heure du déjeuner en fonction du travail à faire. Ils n'aiment pas les horaires fixes, ni se presser. Pour Béatrice, c'est important de rompre avec la cadence de travail imposée au supermarché : "Faut que je sois à 5 heures, faut qu'à 8 beures j'aie fini, faut que je fasse mes commandes avant telle beure, mes machins avant tels jours. C'est un stress perpétuel parce qu'on a toujours dans la tête, bon, faut avoir fini, quelle beure il est, machin. [Alors] qu'avec les bêtes si on a une demi-heure de retard, elles s'en foutent, elles. Y'a pas d'beure." L'élevage lui permet de se détendre: "C'est comme quelqu'un qui va au sauna, prendre une beure de sauna, moi je vais dans la bergerie. "Ils ne comptent pas leur temps en élevage: par exemple, ils donnent beaucoup de biberons aux agneaux, ils sortent les brebis tous les jours en hiver, même pour quelques heures, en triant les agneaux, les allaitantes et les supposées gestantes. Ils ne parlent pas de vacances.

$\rightarrow$ La gestion du temps renvoie surtout à la rationalité relationnelle avec les animaux, à la rationalité relative à l'engagement du corps au travail et très peu aux contraintes horaires.

Le collectif de travail est restreint à eux deux, sauf pour la tonte et pour le tri des agneaux à commercialiser. La répartition des tâches est raisonnée en fonction de leurs goûts et compétences, par exemple à Béatrice les biberons, à Robert les cultures, mais cette répartition tient compte du fait qu'ils aiment travailler ensemble et qu'ils ne veulent confier leurs animaux à d'autres. Ils préfèrent travailler ensemble que gagner du temps en se répartissant le travail avec les bêtes. $\rightarrow$ Le collectif de travail et la répartition des tâches renvoient au vivre ensemble, en couple, et avec les animaux, c'està-dire aux rationalités relationnelles.

Les pratiques sanitaires (vaccins, soins curatifs, euthanasie), pendant les mises bas (allotement, biberon), de réforme (sortir les brebis du troupeau mais les garder sur l'exploitation jusqu'à leur mort), sont exigeantes en temps de travail et ont un coût, mais permettent un investissement affectif important, elles ne sont pas ajustables: "Là, j'ai des biberons, j'en ai 17 sous les lampes... Quand il faut tout soigner, c'est vrai, des fois c'est assez dur... mais ça ne fait rien !... on a 
le contentement quand tout est fait, quand tout va bien."

$\rightarrow$ Les choix de conduite renvoient surtout à la rationalité relationnelle avec les animaux partagée dans le couple et à la rationalité technique de Robert.

Les dimensions de toutes les activités résultent d'ajustements en cours de trajectoire: plus de brebis quand Béatrice est arrivée et un peu moins d'entreprise quand Béatrice a commencé à travailler au supermarché.

$\rightarrow$ Le dimensionnement des activités renvoie à la fois aux rationalités techniques, économiques, relationnelles, liées à l'engagement du corps au travail et à la fois aux contraintes temporelles.

Pour résumer, les choix de conduite et une gestion du temps, qui renvoient aux rationalités relationnelle avec les animaux et technique ainsi que le collectif de travail restreint qui renvoie à la rationalité relationnelle avec les gens, constituent les éléments structurants de l'organisation du travail chez Béatrice et Robert. Le compromis porte sur le sommeil réduit de Béatrice et sa fatigue physique accumulée ainsi que sur la réduction drastique du temps avec les amis : "Moi le soir à 9 beures, il n'y a plus de bonne femme. On ne peut rien faire. [...] Mais c'est un choix pour ça, pour les bêtes. "

\section{Discussion}

Nous discutons le caractère novateur du cadre et la manière de le valider.

Plusieurs travaux soulignent l'importance des dimensions identitaires, affectives, sensibles dans le travail des éleveurs (Salmona, 1994 ; Sens et Soriano, 2001), ainsi que la diversité des modalités d'organisation du travail (Cournut et Hostiou, 2010). Ces approches n'ont cependant jamais été articulées pour construire un regard conjoint sur le travail en élevage. Elles sont en effet séparées par une grande distance théorique. Il y a d'un côté une approche utilitariste :

- du travail, limité à sa dimension productive ;

- de l'éleveur, comme un pilote technico-économique de l'organisation du travail sur l'exploitation, qui intègre les différents impératifs et contraintes matérielles et temporelles et agence les tâches et les travailleurs dans le temps ;
- des choix de l'éleveur selon la rationalité technico-économique (Dedieu et Servière, 2010).

De l'autre côté, il y a une approche antiutilitariste (Caillé, 2009) :

- du travail, comme rapport à la vie, permettant de se construire et de vivre ensemble (Dejours, 1998) ;

- des personnes actives du ménage, comme sujets de leur travail, entretenant chacune dans le travail un rapport à la vie, à soi et aux autres ;

- des choix de ces personnes comme relevant de leurs rationalités subjectives.

C'est pourtant bien dans l'articulation de ces deux approches, qu'émergent les éléments structurants de l'organisation du travail faisant sens pour l'éleveur. Ainsi, par exemple, les choix de conduite, tels que les choix de réforme, de distribuer des biberons sont susceptibles à la fois de porter le sens du travail, identitaire ou relationnel, et de générer la durée et le calendrier de travail.

Notre cadre d'interprétation permet d'interroger de manière originale deux éléments clés du travail en élevage : la diversité des rapports au temps de travail des éleveurs, et la possible indépendance des rationalités techniques et économiques.

L'analyse de cas confirme la diversité des rapports au temps, de l'importance du plaisir et du vivre ensemble au travail dans les choix de consacrer du temps à l'élevage, a contrario d'une vision strictement comptable du travail dans laquelle l'amélioration des conditions de travail est synonyme de maîtrise du temps de travail, d'efficience productive et d'augmentation du temps libre. Comme Blanchemanche (2000) l'illustre en rapportant les propos d'une agricultrice, "le problème du temps et du loisir ça c'est comme je dis souvent ça dépend de ce que l'on fait, si on l'intitule comme du travail ou du plaisir. Moi quand je tonds la pelouse, je me fais plaisir, quand j'accueille mes clients, je me fais plaisir ", les temps de travail et de loisir ne sont pas toujours distincts, ni les temps de travail et en famille. Pour Robert et Béatrice, le temps de l'élevage est du temps avec leurs "filles ", leurs "bébés", en couple, tandis que pour Georges il ne l'est pas. Robert et Béatrice, prennent plaisir à travailler à leur rythme, et non à travailler plus vite pour travailler moins ou de façon plus efficiente. Pour Georges, c'est important d'être relativement efficace sur l'exploitation pour avoir le temps de faire autre chose; en même temps il explique qu'il fait de l'élevage du fait de sa disponibilité liée à son travail de nuit. L'analyse de cas interroge donc :

- le caractère monolithique des revendications de réduction du temps de travail des éleveurs, telles qu'intégrées par l'encadrement agricole et les zootechniciens ;

- le fondement de ces revendications, une vision des temps séparés de travail, en famille, de loisir, et une vision comptable du temps de travail ;

- une vision du travail qui ne tient pas compte du plaisir au travail ;

- mais aussi la pertinence des approches normatives du travail telles que la qualification des équivalents temps complet ou l'estimation du temps disponible, qui s'inspirent de la durée légale du travail salarié comme miroir du rapport au temps des éleveurs.

Le second élément de discussion que suggère notre cadre d'analyse est la possible indépendance entre les rationalités économique et technique. On retrouve ici des distinctions évoquées dans les approches sociologiques des "farming styles" (Van der Ploeg, 1994) qui distinguent le rapport au travail bien fait du "style artisan ", du rapport au travail productif et efficace dans l'optique d'un profit économique maximum du "style entrepreneur".

Notre proposition a certes été développée chez des éleveurs pluriactifs, mais elle nous semble transposable à d'autres situations. En effet, d'autres travaux montrent que l'importance des dimensions identitaires, affectives, sensibles dans le travail des éleveurs (Porcher, 2002 ; Salmona, 1994 ; Sens et Soriano, 2001), ainsi que la diversité des modalités d'organisation du travail (Cournut et Dedieu, 2005), ne sont pas l'apanage des éleveurs pluriactifs.

La validation du cadre d'analyse est en cours. Nous l'avons déjà mis à l'épreuve dans deux arènes : formation d'intervenants auprès d'agriculteurs en difficulté, atelier sur les façons d'être pour aborder le travail dans le conseil (Fiorelli et Couzy, 2009). Les partenaires de ces opérations (techniciens de développement au sens large) ont été intéressés par la réflexivité permise sur leur propre rapport au travail, et par l'explicitation de ce qu'ils nomment la dimension humaine ou personnelle du travail, qu'ils entendent chez les éleveurs mais dont ils ne pouvaient se saisir pour intervenir sur les choix de conduite et d'organisation du travail. 


\section{Conclusion}

Notre cadre d'analyse amène à repenser la façon de représenter l'organisation du travail.

Premièrement, il prend en compte le ressenti de chacun des éleveurs, souligne les faits marquants de leur rapport au travail incluant des aspects identitaires et relationnels et ne se limite pas aux objectifs de production, de revenu ou de calendrier de travail.

Deuxièmement, il permet une analyse de l'organisation du travail des éleveurs dans une perspective compréhensive et non normative, sans se référer à des indicateurs exogènes renseignés exhaustivement, qui n'ont pas forcément de sens pour eux.

Troisièmement, au lieu de caractériser l'organisation réalisée, qui donne à voir une représentation lissée, il met en évidence les compromis, les tensions dans l'organisation du travail et leur sens pour l'éleveur, mais aussi ce que l'éleveur est susceptible de modifier compte tenu de son rapport au travail. Ce faisant, il devrait faciliter l'identification du problème à traiter avec l'éleveur (Magne et Ingrand, 2005) et le passage du diagnostic à la construction de changement, tous deux jugés difficiles par les conseillers (Boissier, 2006).

Ce cadre invite ainsi à penser l'organisation du travail comme l'aménagement par et pour l'éleveur de ses " conditions de vie au travail " (Porcher, 2003).

\section{Références}

Barlett PF. Part-time farming : Saving the farm or saving the life-style? Rural Sociol 1986; 51 : 289-313.

Bessant KC. Part-time farming situations among Manitoba farm operators: a typological approach. Can J Agr Econ 2000 ; 48 : 259-77.

Blanchemanche S. La combinaison d'activités professionnelles des ménages agricoles. L'exemple du département de I'Isère. Paris: Université de Paris X, 2000.
Boissier M. Rapport final de synthèse : Organisation du travail et gestion des ressources humaines. Paris: Vivéa, 2006. www.vivea.fr/

Caillé A. Théorie anti-utilitariste de l'action, fragments d'une sociologie générale. Paris : éditions La Découverte, 2009.

Collectif. Recueil d'outils et démarches de conseil sur le travail en élevage. Troisièmes Rencontres nationales des acteurs du développement, de la recherche et de la formation Travail en élevage, Rennes, 19 et 20 novembre 2009. www.inst-elevage.asso.fr/html1/spip.php? article 16797

Coquillard $\mathrm{P}$, Hill DRC. Modélisation et simulations d'écosystèmes. Paris: éditions Masson, 1997.

Cournut S, Dedieu B. Simplification des conduites d'élevage en bovins laitiers. Cah Agric 2005 ; $14: 541-7$.

Cournut S, Hostiou N. Adaptations des systèmes bovin laitier pour réduire la contrainte travail: une étude en Ségala (France). Cah Agric 2010 ; 19 : epub. doi : 10.1684 /agr.2010.0418

David A. Études de cas et généralisation scientifique en sciences de gestion. Treizième conférence internationale de management stratégique, Association internationale de management stratégique, AIMS, Le Havre, 1-4 juin 2004. www.strategie-aims.com/Normandie04/ sessions/David.pdf

Dedieu B, Chauvat S, Servière G, Tchakerian E. Bilan travail pour l'étude du fonctionnement des exploitations d'élevage: Méthode d'analyse et documents d'enquête. Paris: Institut de l'élevage, 2000. www.inst-elevage.asso.fr

Dedieu B, Laurent C, Mundler P. Organisation du travail dans les systèmes d'activités complexes. Economie rurale 1999; 253: 28-35.

Dedieu B, Servière G, Madelrieux S, Dobremez L, Cournut S. Comment appréhender conjointement les changements techniques et les changements du travail en élevage ? Cah Agric 2006; 15: 506-13. doi : 10.1684/agr.2006.0028

Dedieu B, Servière G. Les modèles du travail en élevage: points de vue de zootechniciens des systèmes d'élevage. Paris : éditions L'Harmattan, (à paraître en 2010).

Dejours C. Travailler n'est pas déroger. Travailler $1998 ; 1: 5-12$

Fagon J, Hostiou N. Simplification des conduites d'élevage: analyse transversale des techniques mises en cuvre dans les filières herbivores et granivores. Troisièmes Rencontres nationales des acteurs du développement, de la recherche et de la formation Travail en élevage, Rennes, 19 et 20 novembre 2009. www.inst-elevage. asso.fr/html1/spip.php?article16797
Fiorelli C, Couzy C. Aborder le travail: partage d'expériences sur les façons d'être. Troisièmes Rencontres nationales des acteurs du développement, de la recherche et de la formation Travail en élevage, Rennes, 19 et 20 novembre 2009. www. inst-elevage.asso.fr/html1/spip.php?article16797

Fiorelli C, Dedieu B, Pailleux JY. Explaining diversity of livestock-farming management strategies of multiple-job holders: importance of level of production objectives and role of farming in the household. Animal 2007a; 1 : 1209-18.

Fiorelli C, Porcher J, Dedieu B. Pourquoi faire de l'élevage quand on a un autre travail ? Quatorzièmes Renc Rech Rum, Paris, 5-6 décembre 2007. 2007b. www.journees3r.fr/

Kling-Eveillard F. Compte rendu de six expériences locales d'accompagnement des éleveurs sur le travail. Paris: Institut de l'élevage, 2008. www.inst-elevage.asso.fr

Macombe C. Work: A necessary sacrifice or a suffered chore? Labor and farm continuity in alternative agriculture in France. Renewable Agriculture and Food Systems 2007 ; 22 : 282-9.

Madelrieux S, Dedieu B, Dobremez L, Girard N. Patterns of work organisation in livestock farms: The ATELAGE approach. Livest Sci 2009; 121 : 28-37.

Magne MA, Ingrand S. Advising beef cattle farmers: problem finding rather than problem solving. Characterization of advice practices in Creuse. J Agric Educ Ext 2005; 10 : 171-9.

Mouret S, Porcher J. Les systèmes industriels porcins: la mort comme travail ordinaire. NSS $2007 ; 15: 245-52$

Porcher J. Bien-être et souffrance en élevage: conditions de vie au travail des personnes et des animaux. Sociol Trav 2003; 45: 27-43.

Porcher J. Éleveurs et animaux: réinventer le lien. Paris: PUF, 2002.

Salmona M. Les paysans français: le travail, les métiers, la transmission des savoirs. Paris: éditions L'Harmattan, 1994.

Seegers J, Moreau JC, Béguin E, Guillaumin A, Frappat B. Attentes des éleveurs laitiers vis-à-vis de leurs conditions de travail et évolution de leurs systèmes d'exploitation. Fourrages 2006 ; 185 : 3-16.

Sens S, Soriano V. Parlez-moi d'élevage. Analyse de représentations d'éleveurs. Dijon: Educagri éditions, 2001

Van der Ploeg JD. Styles of farming. An introductory note on concepts and methodology. In : Van der Ploeg JD, Long A, eds. Born from within. Practices and perspectives on endogenous rural development. Assen : Maastrich (the Netherlands) : Van Gorcum, 1994. 